\title{
DISTRIBUTION OF LENGTH SPECTRUM OF CIRCLES ON A COMPLEX HYPERBOLIC SPACE
}

\author{
TOSHIAKI ADACHI ${ }^{1}$
}

\begin{abstract}
It is well-known that all geodesics on a Riemannian symmetric space of rank one are congruent each other under the action of isometry group. Being concerned with circles, we also know that two closed circles in a real space form are congruent if and only if they have the same length. In this paper we study how prime periods of circles on a complex hyperbolic space are distributed on a real line and show that even if two circles have the same length and the same geodesic curvature they are not necessarily congruent each other.
\end{abstract}

\section{$\S 1$. Introduction}

The aim of this paper is to study the distribution of the length of closed circles on a complex hyperbolic space. In our recent works we gave a light on geometric properties of circles on a Kähler manifold. For example, we interpreted holomorphic circles in terms of magnetic fields ([1], [3]). We defined Kähler magnetic flows associated to these circles, and showed that Kähler magnetic flows of small geodesic curvature on a complex hyperbolic space are conjugate to the geodesic flow, hence are of Anosov type. This result gives us information on length spectrum of holomorphic circles of small geodesic curvature on a compact manifold of constant holomorphic sectional curvature: The number of closed holomorphic circles grows exponentially with respect to their length (cf. [18], [8], [14] for more detail). In this paper we add another result on the feature of the length spectrum of circles of large geodesic curvature on a complex hyperbolic space in connection with the action of the isometry group.

A smooth curve $\gamma: \mathbb{R} \longrightarrow M$ on a complete Riemannian manifold $M$ is called a circle of geodesic curvature $\kappa(\geq 0)$ if it is parametrized by its arc-length and satisfies the following equation:

$$
\nabla_{t} \nabla_{t} \dot{\gamma}(t)=-\kappa^{2} \dot{\gamma}(t)
$$

Received February 20, 1997.

Revised January 7, 1998.

${ }^{1}$ The author partially supported by Grant-in-Aid for Encouragement of Young Scientists (No. 09740055), Ministry of Education, Science and Culture 
where $\nabla_{t}$ denotes the covariant differentiation along $\gamma$ with respect to the Riemannian connection of $M$ ([17]). Needless to say, circles are classified by their geodesic curvature. When $\kappa=0$, this equation is nothing but the equation of geodesics. One may think that the notion of circles is just a natural extension of the notion of geodesics. But when $M$ is a Kähler manifold we have another tool for classification of circles which is associated with the complex structure $J$ : For a circle $\gamma$ we define its complex torsion $\tau$ by $\left\langle\dot{\gamma}, J \nabla_{t} \dot{\gamma}\right\rangle /\left\|\nabla_{t} \dot{\gamma}\right\|$. This does not depend on $t$ and satisfies $|\tau| \leq 1$. It might be natural to think that some properties of circles are related to the Kähler geometry of the base manifold.

We call a circle $\gamma$ closed if there exists a constant $T$ with

$$
\gamma(T)=\gamma(0), \dot{\gamma}(T)=\dot{\gamma}(0), \nabla_{t} \dot{\gamma}(T)=\nabla_{t} \dot{\gamma}(0) .
$$

This condition is equivalent to the condition that $\gamma(t+T)=\gamma(t)$ for every $t$. The minimum positive constant with these properties is called the prime period of $\gamma$ and is denoted by length $(\gamma)$. We put length $(\gamma)=\infty$ for an open circle $\gamma$, a circle which is not closed. We are interested in how prime periods of closed circles are distributed on the real line. It is well known that on a compact rank one symmetric space every geodesic is closed and has the same length. Moreover all these geodesics are congruent each other, and the same thing holds for geodesics on a rank one symmetric space of noncompact type. Here we call two circles $\gamma_{1}$ and $\gamma_{2}$ are congruent if there exist an isometry $\varphi$ and a constant $t_{0}$ with $\gamma_{2}(t)=\varphi \circ \gamma_{1}\left(t+t_{0}\right)$ for every $t$. Being concerned with circles, we have that on a real space form two closed circles are congruent each other if and only if they have the same prime periods. At this stage we are naturally come to have the following problems on circles on a rank one Kähler symmetric space:

1) Is it true that closed circles (of geodesic curvature $\kappa$ ) with the same length are congruent each other?

2 ) If it is not true, then how many congruency classes are there with the same length?

In order to attack these problems we shall consider the moduli space $\operatorname{Cir}(M)$ of circles, which is the quotient space of the set of all circles on $M$ under the congruence relation. The length spectrum of circles on $M$ is the map $\mathcal{L}: \operatorname{Cir}(M) \longrightarrow \mathbb{R} \cup\{\infty\}$ defined by $\mathcal{L}([\gamma])=\operatorname{length}(\gamma)$. Sometimes we also call the image $\operatorname{LSpec}(M)=\mathcal{L}(\operatorname{Cir}(M)) \cap \mathbb{R}$ in the real line the length spectrum of circles on $M$. On a non-flat complex space form, two circles are congruent if and only if they have the same geodesic curvature and the same absolute value of complex torsion (see [15]). The moduli space of circles on a non-flat complex space form hence has canonical foliated structures. So 
we also consider the length spectrum of circles of given geodesic curvature and that of given complex torsion.

In the preceding paper [5] we already investigated the length spectrum of circles on a complex projective space. Hence we here have to treat the length spectrum of circles on a complex hyperbolic space, another model space for Kähler geometry. In treating circles we find a difference between their feature on complex hyperbolic spaces and their feature on complex projective spaces: Circles on a complex hyperbolic space with small geodesic curvature are unbounded open curves. This should reflect on the structure of the length spectrum. In this paper, we first bring our attention to circles of sufficiently large geodesic curvature. On a complex hyperbolic space $\mathbb{C} H^{n}(-c)$ of holomorphic sectional curvature $-c$, every circle with geodesic curvature $\kappa>\sqrt{c}$ is bounded. In section 2 , we compare by a map of duality the structure of the length spectrum of circles of geodesic curvature $\kappa(>\sqrt{c})$ on a complex hyperbolic space with that on a complex projective space. Once a map of duality was constructed, some arguments in [5] go through on our case. We show the following in section 3.

THEOREM 1. On a complex hyperbolic space $\mathbb{C} H^{n}(-c)$ of holomorphic sectional curvature $-c$,

1) we can not distinguish congruency classes of circles of geodesic curvature $\kappa$ by their length spectrum.

2) The number of congruency classes of circles of geodesic curvature $\kappa$ with length $\lambda$ is not bounded with respect to $\lambda$.

The unbounded property of circles of small geodesic curvature reflects on internal properties of length spectrum. With the aid of the map of normalization constructed in section 2, we obtain a natural foliation on the moduli space of bounded circles. This foliation leads us to many nice results on the length spectrum. On a compact projective space, the circle with minimal length in the set of circles of curvature $\kappa$ is a holomorphic circle, a circle with complex torsion \pm 1 , and the circle with second minimal length is a totally real circle, a circle with null complex torsion. Contrary, we have the following:

TheOREM 2. On a complex hyperbolic space $\mathbb{C} H^{n}(-c)$,

1) totally real circles have the minimal length in the set of circles of geodesic curvature $\kappa$,

2) holomorphic circles have the second minimal length in the set of circles of geodesic curvature $\kappa$ if and only if $\kappa \geq \frac{5 \sqrt{c}}{4}$. 
The unbounded property also reflects on the structure of the full length spectrum LSpec $\left(\mathbb{C} H^{n}(-c)\right)$. For a spectrum $\lambda \in \operatorname{LSpec}\left(\mathbb{C} H^{n}(-c)\right)$ we call the cardinality $\sharp\left(\mathcal{L}^{-1}(\lambda)\right)$ of the set $\mathcal{L}^{-1}(\lambda)$ the multiplicity of $\mathcal{L}$ at $\lambda$. The multiplicity indicates the number of congruency classes of circles with length $\lambda$. When the multiplicity is one, we shall call this spectrum simple. Different from the case of complex projective spaces, we trivially find that there is no simple spectrum for $\mathcal{L}$. In particular, we find no congruency classes of circles can be distinguished only by the length spectrum. We show in section 4 the following result.

\section{TheOREM 3.}

1) The length spectrum LSpec $\left(\mathbb{C} H^{n}(-c)\right)$ of circles on $\mathbb{C} H^{n}(-c)$ coincides with the half line $(0, \infty)$.

2) The multiplicity of length spectrum $\mathcal{L}$ is finite and greater than one at each point $\lambda \in \mathbb{R}$, and is monotone increasing.

3) There are no circles $\gamma$ on $\mathbb{C} H^{n}(-c)$ of complex torsion $\tau(0<|\tau|<1)$ with $\operatorname{length}(\gamma) \leq \frac{8}{3 \sqrt{c}} \pi$.

In the final section we also study the length spectrum of circles of a given complex torsion. With the study of structure of the natural foliation, we give an estimate of the first length spectrum in these. For the sake of readers' convenience we give tables which show the difference between the length spectrum of circles on a complex hyperbolic space and that on a complex projective space. But the reader should refer [5]. Some proofs which follow directly from [5] were left out.

The author would like to thank Professor Masakazu Yamagishi for valuable advice on classical number theory.

\section{$\S 2$. Canonical transformations on the moduli space}

In this section we study some fundamental properties on the length spectrum of circles of geodesic curvature $\kappa$ on a complex hyperbolic space $\mathbb{C} H^{n}(-c)$ of holomorphic sectional curvature $-c$. In the preceding paper [4] we showed the following on prime periods of circles on a complex hyperbolic space.

FACT 1. ([4]) For given $\tau(|\tau| \leq 1)$ we denote by $\kappa(\tau)$ the unique positive solution for the equation

$$
\left(4 \kappa^{2}-c\right)^{3 / 2}-3 \sqrt{3} c \kappa \tau=0 .
$$

Circles on $\mathbb{C} H^{n}(-c)$ have the following properties: 
1) Every circle of complex torsion $\tau$ and geodesic curvature $\kappa \leq \kappa(\tau)$ is a unbounded open curve.

2) Every holomorphic circle of geodesic curvature $\kappa>\sqrt{c}$ is closed with prime period $\frac{2 \pi}{\sqrt{\kappa^{2}-c}}$.

3) Every totally real circle of geodesic curvature $\kappa>\frac{\sqrt{c}}{2}$ is closed with prime period $\frac{4 \pi}{\sqrt{4 \kappa^{2}-c}}$.

4) When $\kappa>\kappa(\tau)$ and $\tau \neq \pm 1$ we denote by $a_{\kappa, \tau}, b_{\kappa, \tau}, d_{\kappa, \tau}\left(a_{\kappa, \tau}<b_{\kappa, \tau}<\right.$ $\left.d_{\kappa, \tau}\right)$ the solutions for the cubic equation

$$
c \lambda^{3}-\left(4 \kappa^{2}-c\right) \lambda+2 \sqrt{c} \kappa \tau=0 .
$$

$A$ circle $\gamma$ of geodesic curvature $\kappa$ and complex torsion $\tau(\neq 0, \pm 1)$ on $\mathbb{C} H^{n}(-c)$ is closed if and only if one of the ratios $a_{\kappa, \tau} / b_{\kappa, \tau}, b_{\kappa, \tau} / d_{\kappa, \tau}$, $d_{\kappa, \tau} / a_{\kappa, \tau}$ is rational. Its prime period is

$$
\operatorname{length}(\gamma)=\frac{4 \pi}{\sqrt{c}} \cdot \text { L.C.M. }\left(\frac{1}{b_{\kappa, \tau}-a_{\kappa, \tau}}, \frac{1}{d_{\kappa, \tau}-a_{\kappa, \tau}}\right)
$$

Here, for two real numbers $\alpha, \beta$, the least common multiple L.C.M. $(\alpha, \beta)$ is the minimum value of the set $\{\alpha n \mid n=1,2,3, \cdots\} \cap\{\beta n \mid n=1,2,3, \cdots\}$.

On a complex hyperbolic space two circles are congruent if and only if they have the same geodesic curvature and the same absolute value of complex torsion. Therefore the moduli space $\operatorname{Cir}\left(\mathbb{C} H^{n}(-c)\right)$ of circles on $\mathbb{C} H^{n}(-c)$ is bijective to the set $[0, \infty) \times[0,1] / \sim$, where $(\kappa, \tau)$ and $\left(\kappa^{\prime}, \tau^{\prime}\right)$ are equivalent if and only if $(\kappa, \tau)=\left(\kappa^{\prime}, \tau^{\prime}\right)$ or $\kappa=\kappa^{\prime}=0$. We denote by $\left[\gamma_{\kappa, \tau}\right]$ the congruency class of circles of geodesic curvature $\kappa$ and complex torsion $\tau(\geq 0)$ on $\mathbb{C} H^{n}(-c)$. The moduli space $\operatorname{Cir}_{\kappa}\left(\mathbb{C} H^{n}(-c)\right)$ of circles of geodesic curvature $\kappa$ hence is the set $\left\{\left[\gamma_{\kappa, \tau}\right] \mid 0 \leq \tau \leq 1\right\}$. By Fact 1 , we find that every circles on $\mathbb{C} H^{n}(-c)$ of geodesic curvature $\kappa$ is unbounded when $\kappa \leq \sqrt{c} / 2$ and is bounded when $\kappa>\sqrt{c}$. To study the length spectrum of circles, we are enough to treat bounded circles. We define a nonnegative function $\nu:[0, \infty) \rightarrow \mathbb{R}$ by

$$
\nu(\kappa)= \begin{cases}0, & \text { if } 0 \leq \kappa<\frac{\sqrt{c}}{2}, \\ \frac{\left(4 \kappa^{2}-c\right)^{3 / 2}}{3 \sqrt{3} c \kappa}, & \text { if } \frac{\sqrt{c}}{2} \leq \kappa \leq \sqrt{c} \\ 1, & \text { if } \kappa>\sqrt{c} .\end{cases}
$$


We then get that circles with geodesic curvature $\kappa$ and complex torsion $\tau<\nu(\kappa)$ is bounded. We put $\mathcal{M}_{\kappa}=\left\{\left[\gamma_{\kappa, \tau}\right] \mid 0 \leq \tau<\nu(\kappa)\right\}$, and define a map of normalization

$$
\Phi_{\kappa}: \mathcal{M}_{\kappa} \longrightarrow \mathcal{M}_{\sqrt{c}}=\operatorname{Cir}_{\sqrt{c}}\left(\mathbb{C} H^{n}(-c)\right) \backslash\left\{\left[\gamma_{\sqrt{c}, 1}\right]\right\}
$$

by

$$
\Phi_{\kappa}\left(\left[\gamma_{\kappa, \tau}\right]\right)=\left[\gamma_{\sqrt{c}, \frac{3 \sqrt{3} c \kappa \tau}{\left(4 \kappa^{2}-c\right)^{3 / 2}}}\right]
$$

LEMma 1. The map of normalization $\Phi_{\kappa}$ is injective and satisfies

$$
\mathcal{L}\left(\left[\gamma_{\kappa, \tau}\right]\right)=C_{\kappa} \cdot \mathcal{L} \circ \Phi_{\kappa}\left(\left[\gamma_{\kappa, \tau}\right]\right), \quad\left[\gamma_{\kappa, \tau}\right] \in \mathcal{M}_{\kappa}
$$

with $C_{\kappa}=\sqrt{\frac{3 c}{4 \kappa^{2}-c}}$. When $\frac{\sqrt{c}}{2}<\kappa<\sqrt{c}$, this map $\Phi_{\kappa}$ is bijective.

Proof. Consider the cubic equation $c \lambda^{3}-\left(4 \kappa^{2}-c\right) \lambda+2 \sqrt{c} \kappa \tau=0$. By putting $\Lambda=C_{\kappa} \lambda$ we find it is equivalent to $c \Lambda^{3}-3 c \Lambda+2 \sqrt{c} \kappa \tau C_{\kappa}^{3}=0$. Since $0 \leq \frac{\kappa \tau}{\sqrt{c}} C_{\kappa}^{3}=3 \sqrt{3} c \kappa \tau\left(4 \kappa^{2}-c\right)^{-3 / 2}<1$, this means that

$$
a_{\sqrt{c}, \mu}=C_{\kappa} a_{\kappa, \tau}, \quad b_{\sqrt{c}, \mu}=C_{\kappa} b_{\kappa, \tau}, \quad d_{\sqrt{c}, \mu}=C_{\kappa} d_{\kappa, \tau},
$$

with $\mu=3 \sqrt{3} c \kappa \tau\left(4 \kappa^{2}-c\right)^{-3 / 2}$. We hence get the conclusion with Fact 1 .

This lemma shows the relation of the structures of the length spectrum of circles of given geodesic curvatures. Let $\mathcal{L}_{\kappa}$ denote the length spectrum of circles of geodesic curvature $\kappa$, which is the restriction of $\mathcal{L}$ onto the moduli space $\operatorname{Cir}_{\kappa}\left(\mathbb{C} H^{n}(-c)\right)$. We shall call $\mathcal{L}_{\kappa_{1}}$ and $\mathcal{L}_{\kappa_{2}}$ are equivalent if there exist a bijection $\Phi_{\kappa_{2}, \kappa_{1}}: \mathrm{Cir}_{\kappa_{1}}\left(\mathbb{C} H^{n}(-c)\right) \rightarrow \mathrm{Cir}_{\kappa_{2}}\left(\mathbb{C} H^{n}(-c)\right)$ and a positive constant $C_{\kappa_{1}, \kappa_{2}}$ with $\mathcal{L}_{\kappa_{1}}=C_{\kappa_{1}, \kappa_{2}} \cdot \mathcal{L}_{\kappa_{2}} \circ \Phi_{\kappa_{2}, \kappa_{1}}$. Since $\operatorname{Cir}_{\kappa}\left(\mathbb{C} H^{n}(-c)\right) \backslash$ $\left(\mathcal{M}_{\kappa} \cup\left\{\left[\gamma_{\kappa, 1}\right]\right\}\right)$ is the set of congruency classes of unbounded circles of geodesic curvature $\kappa$, Lemma 1 guarantees that the length spectrum $\mathcal{L}_{\kappa}$ is essentially equivalent to $\mathcal{L}_{\sqrt{c}}$ if $\frac{\sqrt{c}}{2}<\kappa<\sqrt{c}$. As a direct consequence of this, defining a bijection $\Phi_{\kappa_{2}, \kappa_{1}}: \operatorname{Cir}_{\kappa_{1}}\left(\mathbb{C} H^{n}(-c)\right) \rightarrow \operatorname{Cir}_{\kappa_{2}}\left(\mathbb{C} H^{n}(-c)\right)$ for $\frac{\sqrt{c}}{2}<\kappa_{1}, \kappa_{2}<\sqrt{c}$ by

$$
\Phi_{\kappa_{2}, \kappa_{1}}\left(\left[\gamma_{\kappa_{1}, \tau}\right]\right)= \begin{cases}\Phi_{\kappa_{2}}^{-1} \circ \Phi_{\kappa_{1}}\left(\left[\gamma_{\kappa_{1}, \tau}\right]\right), & 0 \leq \tau<\nu\left(\kappa_{1}\right), \\ {\left[\gamma_{\kappa_{2}, 1-\frac{1-\nu\left(\kappa_{2}\right)}{1-\nu\left(\kappa_{1}\right)}(1-\tau)}\right],} & \nu\left(\kappa_{1}\right) \leq \tau \leq 1,\end{cases}
$$

we get the following: 
Proposition 1. On a complex hyperbolic space $\mathbb{C} H^{n}(-c)$,

1) the length spectrum $\mathcal{L}_{\kappa}\left(\frac{\sqrt{c}}{2}<\kappa \leq \sqrt{c}\right)$ are equivalent each other,

2) when $\kappa>\sqrt{c}$, the length spectrum $\mathcal{L}_{\kappa}$ is not equivalent to any $\mathcal{L}_{\kappa^{\prime}}$.

On a complex projective space $\mathbb{C} P^{n}(c)$ of holomorphic sectional curvature $c$, for any positive $\kappa$ the length spectrum $\mathcal{L}_{\kappa}$ is equivalent only to $\mathcal{L}_{\kappa^{\prime}}$, where $\kappa^{\prime}(\neq \kappa)$ is the unique positive number with $\frac{\kappa^{\prime 2}}{\left(4 \kappa^{\prime 2}+c\right)^{3}}=\frac{\kappa^{2}}{\left(4 \kappa^{2}+c\right)^{3}}$.

Following Lemma 1 we set

$$
\mathcal{F}_{\mu}= \begin{cases}\left\{\left[\gamma_{\kappa, 0}\right] \mid \kappa>\frac{\sqrt{c}}{2}\right\}, & \text { if } \mu=0, \\ \left\{\left[\gamma_{\kappa, \tau}\right] \mid 3 \sqrt{3} c \kappa \tau\left(4 \kappa^{2}-c\right)^{-3 / 2}=\mu, 0<\tau<1\right\}, & \text { if } 0<\mu<1, \\ \left\{\left[\gamma_{\kappa, 1}\right] \mid \kappa>\sqrt{c}\right\}, & \text { if } \mu=1 .\end{cases}
$$

This gives a natural foliation of the moduli space

$$
\operatorname{BCir}\left(\mathbb{C} H^{n}(-c)\right)=\left\{\left[\gamma_{\kappa, \tau}\right] \mid \kappa>\kappa(\tau), 0 \leq \tau \leq 1\right\}
$$

of bounded circles on a complex hyperbolic space.

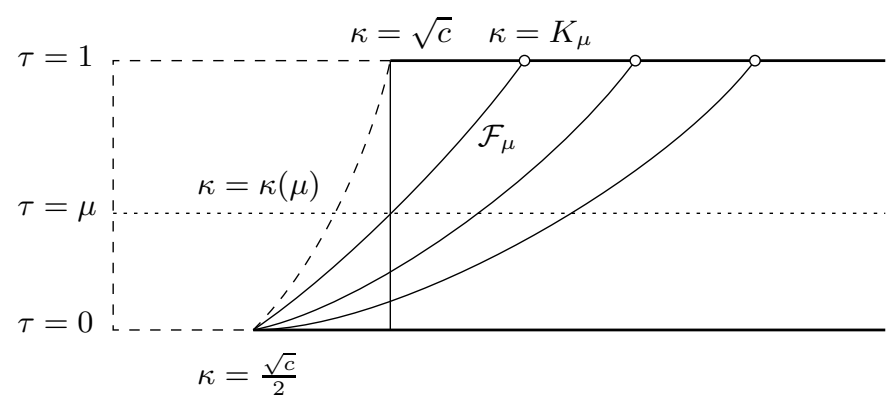

Figure (Foliation of the moduli space of bounded circles on $\mathbb{C} H^{n}(-c)$ )

We now compare the length spectrum of circles on a complex hyperbolic space with that on a complex projective space $\mathbb{C} P^{n}(c)$. We define a map of duality

$$
\Psi: \operatorname{Cir}_{\sqrt{c}}\left(\mathbb{C} H^{n}(-c)\right) \backslash\left\{\left[\gamma_{\sqrt{c}, 1}\right]\right\} \longrightarrow \operatorname{Cir}_{\frac{\sqrt{2 c}}{4}}\left(\mathbb{C} P^{n}(c)\right) \backslash\left\{\left[\gamma_{\frac{\sqrt{2 c}}{4}, 1}\right]\right\}
$$

by $\Psi\left(\left[\gamma_{\sqrt{c}, \tau}\right]\right)=\left[\gamma_{\frac{\sqrt{2 c}}{4}, \tau}\right]$. Here we use the same notation $\left[\gamma_{\kappa, \tau}\right]$ for representing the congruency class of circles on $\mathbb{C} P^{n}(c)$ with geodesic curvature $\kappa$ and complex torsion $\tau$. 
FACT 2. (cf. [5] and [6]) On a complex projective space $\mathbb{C} P^{n}(c)$ of holomorphic sectional curvature $c$, a circle $\gamma$ of geodesic curvature $\sqrt{2 c} / 4$ and complex torsion $\tau(0<|\tau|<1)$ satisfies the following properties:

1) Let $A, B$, and $D(A<B<D)$ denotes the solutions for the cubic equation

$$
\lambda^{3}-\frac{3}{2} \lambda+\frac{\sqrt{2}}{2} c \kappa \tau=0 .
$$

The circle $\gamma$ is closed if and only if one of the ratios $A / B, B / D, D / A$ is rational. Its prime period is

$$
\operatorname{length}(\gamma)=\frac{4 \pi}{\sqrt{c}} \text {. L.C.M. }\left(\frac{1}{B-A}, \frac{1}{D-A}\right)
$$

2) The circle $\gamma$ is closed if and only if the complex torsion $\tau$ is of the form

$$
\tau=\tau(p, q)=q\left(9 p^{2}-q^{2}\right)\left(3 p^{2}+q^{2}\right)^{-3 / 2}
$$

for some mutually prime positive integers $p, q$ with $p>q$. In this case its prime period is

$$
\text { length }(\gamma)= \begin{cases}\frac{4}{3 \sqrt{c}} \pi \sqrt{2\left(3 p^{2}+q^{2}\right)}, & \text { if } p q \text { is even, } \\ \frac{2}{3 \sqrt{c}} \pi \sqrt{2\left(3 p^{2}+q^{2}\right)}, & \text { if } p q \text { is odd. }\end{cases}
$$

By the same argument as in the proof of Lemma 1 we obtain the following:

LEMMA 2. The map of duality $\Psi$ satisfies

$$
\mathcal{L}\left(\left[\gamma_{\sqrt{c}, \tau}\right]\right)=\frac{1}{\sqrt{2}} \cdot \mathcal{L} \circ \Psi\left(\left[\gamma_{\sqrt{c}, \tau}\right]\right)
$$

for every $\tau(0 \leq \tau<1)$.

\section{$\S 3$. The length spectrum of circles of geodesic curvature $\kappa$}

We study in this section the length spectrum of circles with a given geodesic curvature. The multiplicity of the length spectrum $\mathcal{L}_{\kappa}$ at $\lambda$ shows the number of congruency classes of circles with geodesic curvature $\kappa$ and length $\lambda$. So our problems mentioned in the introduction can be put into the problems on the multiplicity of $\mathcal{L}_{\kappa}$. 
Put $\operatorname{LSpec}_{\kappa}\left(\mathbb{C} H^{n}(-c)\right)=\mathcal{L}\left(\operatorname{Cir}_{\kappa}\left(\mathbb{C} H^{n}(-c)\right)\right) \cap \mathbb{R}$. As a direct consequence of Lemma 2, Facts 1 and 2 we have

$$
\begin{aligned}
& \operatorname{LSpec}_{\sqrt{c}}\left(\mathbb{C} H^{n}(-c)\right) \\
& =\left\{\frac{4 \pi}{\sqrt{3 c}}\right\} \bigcup\left\{\frac{4}{3 \sqrt{c}} \pi \sqrt{3 p^{2}+q^{2}} \mid \begin{array}{l}
p>q, p q \text { is even, } \\
p \text { and } q \text { are mutually prime }
\end{array}\right\} \\
& \bigcup\left\{\frac{2}{3 \sqrt{c}} \pi \sqrt{3 p^{2}+q^{2}} \mid \begin{array}{l}
p>q, p q \text { is odd, } \\
p \text { and } q \text { are mutually prime }
\end{array}\right\} \\
& =\left\{\frac{4 \pi}{\sqrt{3 c}}, \frac{4 \pi}{3 \sqrt{c}} \sqrt{7}, \frac{4 \pi}{3 \sqrt{c}} \sqrt{13}, \frac{4 \pi}{3 \sqrt{c}} \sqrt{19}, \frac{4 \pi}{3 \sqrt{c}} \sqrt{21},\right. \\
& \frac{4 \pi}{3 \sqrt{c}} \sqrt{31}, \frac{4 \pi}{3 \sqrt{c}} \sqrt{37}, \frac{4 \pi}{3 \sqrt{c}} \sqrt{39}, \frac{4 \pi}{3 \sqrt{c}} \sqrt{43}, \frac{28 \pi}{3 \sqrt{c}}, \frac{4 \pi}{3 \sqrt{c}} \sqrt{57}, \\
& \frac{4 \pi}{3 \sqrt{c}} \sqrt{61}, \frac{4 \pi}{3 \sqrt{c}} \sqrt{67}, \frac{4 \pi}{3 \sqrt{c}} \sqrt{73}, \frac{4 \pi}{3 \sqrt{c}} \sqrt{78}, \frac{4 \pi}{3 \sqrt{c}} \sqrt{79}, \\
& \left.\frac{4 \pi}{3 \sqrt{c}} \sqrt{91}, \frac{4 \pi}{3 \sqrt{c}} \sqrt{93}, \frac{4 \pi}{3 \sqrt{c}} \sqrt{97}, \frac{4 \pi}{3 \sqrt{c}} \sqrt{109}, \frac{4 \pi}{3 \sqrt{c}} \sqrt{111}, \cdots\right\} .
\end{aligned}
$$

This shows that we can not distinguish two congruency classes of circles of geodesic curvature $\sqrt{c}$ on a complex hyperbolic space only by their length spectrum. For example, $\frac{4 \pi}{3 \sqrt{c}} \sqrt{91}$ is the smallest double length spectrum for circles of geodesic curvature $\sqrt{c}$. This spectrum is the length of circles of complex torsion $\tau(11,1)=\frac{135}{91 \sqrt{91}}$ and $\tau(5,4)=\frac{836}{91 \sqrt{91}}$ with the geodesic curvature $\sqrt{c}$. The second double length spectrum is $\frac{4 \pi}{3 \sqrt{c}} \sqrt{133}$, which is the length of circles of complex torsion $\tau(6,5)$ and $\tau(13,5)$. The multiplicity of the length spectrum $\mathcal{L}_{\sqrt{c}}$ at $\frac{4 \pi}{3 \sqrt{c}} \sqrt{13741}$ is at least 3 . This spectrum is the length of circles of complex torsion $\tau(129,71), \tau(131,59)$ and $\tau(135,17)$. Moreover, by a classical number theory, we find that the number of congruency classes of circles of geodesic curvature $\sqrt{c}$ with length $\lambda$ is not uniformly bounded with respect to $\lambda$; $\lim \sup _{\lambda \rightarrow \infty} \sharp\left(\mathcal{L}_{\sqrt{c}}^{-1}(\lambda)\right)=\infty$ (see for example Chapter II of [11] or Theorem 278 (p. 242) of [13]). On the order of growth, we also obtain

$$
\lim _{\lambda \rightarrow \infty}(\log \lambda)^{-\alpha_{\sharp}} \sharp\left(\mathcal{L}_{\sqrt{c}}^{-1}(\lambda)\right)=0 \quad \text { for every } \alpha>0 .
$$

We now consider for general $\kappa$. When $\kappa \leq \frac{\sqrt{c}}{2}$, since every circle of geodesic curvature $\kappa$ is open, it is clear that $\operatorname{LSpec}_{\kappa}\left(\mathbb{C} H^{n}(-c)\right)$ is an empty 
set. For $\kappa>\frac{\sqrt{c}}{2}$, since the map of normalization $\Phi_{\kappa}$ is injective and its image is

$$
\left\{\left[\gamma_{\sqrt{c}, \mu}\right] \mid 0 \leq \mu<\frac{3 \sqrt{3} c \kappa \nu(\kappa)}{\left(4 \kappa^{2}-c\right)^{3 / 2}}\right\},
$$

we obtain the following by recalling the definition of $\nu$ : When $\frac{\sqrt{c}}{2}<\kappa \leq \sqrt{c}$,

$$
\begin{aligned}
\operatorname{LSpec}_{\kappa}\left(\mathbb{C} H^{n}(-c)\right) & \left\{\frac{4 \pi}{\sqrt{4 \kappa^{2}-c}}\right\} \bigcup\left\{4 \pi \sqrt{\frac{3 p^{2}+q^{2}}{3\left(4 \kappa^{2}-c\right)}} \mid \begin{array}{l}
p>q, p q \text { is even, } \\
p \text { and } q \text { are mutually prime }
\end{array}\right\} \\
& \bigcup\left\{2 \pi \sqrt{\frac{3 p^{2}+q^{2}}{3\left(4 \kappa^{2}-c\right)}} \mid \begin{array}{l}
p>q, p q \text { is odd, } \\
p \text { and } q \text { are mutually prime }
\end{array}\right\},
\end{aligned}
$$

and when $\kappa>\sqrt{c}$,

$$
\begin{aligned}
& \operatorname{LSpec}_{\kappa}\left(\mathbb{C} H^{n}(-c)\right) \\
& =\left\{\frac{4 \pi}{\left.\sqrt{4 \kappa^{2}-c}, \frac{2 \pi}{\sqrt{\kappa^{2}-c}}\right\}}\right. \\
& \qquad\left\{4 \pi \sqrt{\frac{3 p^{2}+q^{2}}{3\left(4 \kappa^{2}-c\right)}} \begin{array}{l}
p>\delta_{\kappa} q, p q \text { is even, } \\
p \text { and } q \text { are mutually prime }
\end{array}\right\} \\
& \bigcup\left\{2 \pi \sqrt{\frac{3 p^{2}+q^{2}}{3\left(4 \kappa^{2}-c\right)}} \mid \begin{array}{l}
p>\delta_{\kappa} q, p q \text { is odd, } \\
p \text { and } q \text { are mutually prime }
\end{array}\right\},
\end{aligned}
$$

where $\delta_{\kappa}(>1)$ denotes the number with $\frac{3 \sqrt{3} c \kappa}{\left(4 \kappa^{2}-c\right)^{3 / 2}}=\frac{9 \delta_{\kappa}^{2}-1}{\left(3 \delta_{\kappa}^{2}+1\right)^{3 / 2}}$. We can therefore conclude the following, which includes Theorem 1:

Proposition 2. When $0 \leq \kappa \leq \frac{\sqrt{c}}{2}$, the length spectrum of circles $\operatorname{LSpec}_{\kappa}\left(\mathbb{C} H^{n}(-c)\right)$ of geodesic curvature $\kappa$ is an empty set. When $\kappa>\frac{\sqrt{c}}{2}$, the following properties hold:

1) The length spectrum $\operatorname{LSpec}_{\kappa}\left(\mathbb{C} H^{n}(-c)\right)$ is a discrete subset of $\mathbb{R}$.

2) The bottom of the set $\operatorname{LSpec}_{\kappa}\left(\mathbb{C} H^{n}(-c)\right)$ is $\frac{4 \pi}{\sqrt{4 \kappa^{2}-c}}$, which is the length of totally real circles of geodesic curvature $\kappa$, and is simple.

3) The multiplicity of the length spectrum $\mathcal{L}_{\kappa}$ is not uniformly bounded with respect to $\lambda$; lim $\sup _{\lambda \rightarrow \infty} \sharp\left(\mathcal{L}_{\kappa}^{-1}(\lambda)\right)=\infty$.

4) The growth order of the multiplicity is smaller than the logarithmic order; $\lim _{\lambda \rightarrow \infty}(\log \lambda)^{-\alpha \sharp}\left(\mathcal{L}_{\kappa}^{-1}(\lambda)\right)=0$ for every $\alpha>0$. 
We now concern the second length spectrum. On a complex projective space, the first length spectrum in $\operatorname{LSpec}_{\kappa}\left(\mathbb{C} P^{n}(c)\right)$ is the length of holomorphic circles and the second is the length of totally real circles. On a complex hyperbolic space $\mathbb{C} H^{n}(-c)$, the situation is not the same. For example, when $\kappa=\sqrt{c}$, the second length spectrum is $\frac{4 \pi}{3 \sqrt{c}} \sqrt{7}$, which is the length of circles of complex torsion $\tau(3,1)=\frac{10}{7 \sqrt{7}}$. Here we compare $\frac{2 \pi}{\sqrt{\kappa^{2}-c}}$, the length of a holomorphic circle, and $4 \pi \sqrt{\frac{7}{3\left(4 \kappa^{2}-c\right)}}$, which corresponds to the length of a circle of complex torsion $\frac{\left(4 \kappa^{2}-c\right)^{3 / 2}}{3 \sqrt{3} c \kappa} \tau(3,1)$. The former is smaller than the latter if and only if $\kappa>\frac{5 \sqrt{c}}{4}$. Since $\delta_{\kappa}$ is monotone increasing with respect to $\kappa$, and $\frac{\delta_{5 \sqrt{c}}}{4}=3$, we get that $0<\frac{\left(4 \kappa^{2}-c\right)^{3 / 2}}{3 \sqrt{3} c \kappa} \tau(3,1)<1$ if $\frac{\sqrt{c}}{2}<\kappa<\frac{5 \sqrt{c}}{4}$ (that is, the latter is the length of a circle of this complex torsion). Therefore we find the following:

\section{Proposition 3.}

1) The second length spectrum in $\operatorname{LSpec}_{\kappa}\left(\mathbb{C} H^{n}(-c)\right)$ is simple for any $\kappa$.

2) If $\kappa \geq \frac{5 \sqrt{c}}{4}$ the second length spectrum is $\frac{2 \pi}{\sqrt{\kappa^{2}-c}}$, the length of holomorphic circles. If $\frac{\sqrt{c}}{2}<\kappa<\frac{5 \sqrt{c}}{4}$, the second length spectrum is $4 \pi \sqrt{\frac{7}{3\left(4 \kappa^{2}-c\right)}}$, which is the length of circles of complex torsion $\frac{10\left(4 \kappa^{2}-c\right)^{3 / 2}}{21 \sqrt{21} c \kappa}$.

The third spectrum also has a nice property. Put $r_{n}=\sqrt{\frac{c}{8(3 n-1)}}(3 n+$ $1)$. This satisfies $\left(9 n^{2}-1\right)^{2}\left(4 r_{n}^{2}-c\right)^{3}-27 c^{2}\left(3 n^{2}+1\right)^{3} r_{n}^{2}=0$, which means that it is the unique positive number with $\delta_{r_{n}}=n$. It is monotone increasing with respect to $n$.

\section{Proposition 4.}

1) The third length spectrum in $\operatorname{LSpec}_{\kappa}\left(\mathbb{C} H^{n}(-c)\right)$ is simple for any $\kappa$.

2) If $\frac{\sqrt{c}}{2}<\kappa<r_{2}\left(=\frac{7 \sqrt{10 c}}{20}\right)$, the third length spectrum is $4 \pi \sqrt{\frac{13}{3\left(4 \kappa^{2}-c\right)}}$, which is the length of circles of complex torsion $\frac{\left(4 \kappa^{2}-c\right)^{3 / 2}}{3 \sqrt{3} c \kappa} \tau(3,1)=$ $\frac{10\left(4 \kappa^{2}-c\right)^{3 / 2}}{21 \sqrt{21} c \kappa}$. If $r_{2} \leq \kappa<r_{3}\left(=\frac{5 \sqrt{c}}{4}\right)$, the third length spectrum is $\frac{2 \pi}{\sqrt{\kappa^{2}-c}}$, the length of holomorphic circles.

3) When $r_{2 m-1} \leq \kappa<r_{2 m+1}, m=2,3, \cdots$, the third length spectrum is $4 \pi \sqrt{\frac{3 m^{2}+3 m+1}{3\left(4 \kappa^{2}-c\right)}}$, the length of circles of complex torsion $\frac{\left(4 \kappa^{2}-c\right)^{3 / 2}}{3 \sqrt{3} c \kappa} \times$ $\tau(2 m+1,1)=\frac{9 m^{2}+9 m+2}{6 \sqrt{3} c \kappa} \times\left(\frac{4 \kappa^{2}-c}{3 m^{2}+3 m+1}\right)^{3 / 2}$. 
Proof. We get the second assertion by direct calculation. Since $\delta_{\kappa}$ is monotone increasing with respect to $\kappa$, we have $\delta_{\kappa} \geq 2 m-1$ for $\kappa \geq r_{2 m-1}$. We therefore study the length spectrum

$$
\frac{4 \pi}{\sqrt{3\left(4 \kappa^{2}-c\right)}} \sqrt{3 p^{2}+q^{2}} \quad \text { for } p>(2 m-1) q, p q=\text { even }
$$

and

$$
\frac{2 \pi}{\sqrt{3\left(4 \kappa^{2}-c\right)}} \sqrt{3 p^{2}+q^{2}} \quad \text { for } p>(2 m-1) q, p q=\text { odd }
$$

When $q \geq 2$, we have $p>2(2 m-1)>2 m+1$, hence $3 p^{2}+q^{2}>3(2 m+1)^{2}+1$. When $q=1$, we have $p \geq 2 m$. Since $4\left\{3(2 m)^{2}+1\right\}>3(2 m+1)^{2}+1$, we find that $\frac{2 \pi}{\sqrt{3\left(4 \kappa^{2}-c\right)}} \sqrt{3(2 m+1)^{2}+1}$ is the smallest spectrum in these.

The simpleness does not holds for the forth spectrum in general. By a similar argument as in the proof of Proposition 4, we get the following.

\section{Proposition 5.}

1) The forth length spectrum $\frac{16 \pi}{3 \sqrt{c}}$ of circles in $\operatorname{LSpec}_{\sqrt{73 c} / 8}\left(\mathbb{C} H^{n}(-c)\right)$ is double.

2) Except for the case $\kappa=\frac{\sqrt{73 c}}{8}$, the forth length spectrum of circles in $\operatorname{LSpec}_{\kappa}\left(\mathbb{C} H^{n}(-c)\right)$ is simple.

3) If $\frac{\sqrt{c}}{2}<\kappa \leq \frac{\sqrt{73 c}}{8}$ or if $r_{2}\left(=\frac{7 \sqrt{10 c}}{20}\right) \leq \kappa<r_{3}\left(=\frac{5 \sqrt{c}}{4}\right)$, the forth length spectrum is $4 \pi \sqrt{\frac{19}{3\left(4 \kappa^{2}-c\right)}}$, which is the length of circles of complex torsion $\frac{\left(4 \kappa^{2}-c\right)^{3 / 2}}{3 \sqrt{3} c \kappa} \tau(5,1)=\frac{28\left(4 \kappa^{2}-c\right)^{3 / 2}}{57 \sqrt{57} c \kappa}$.

4) If $\frac{\sqrt{73 c}}{8} \leq \kappa<r_{2}$, the forth length spectrum is $\frac{2 \pi}{\sqrt{\kappa^{2}-c}}$, the length of holomorphic circles.

5) When $r_{2 m-1} \leq \kappa<r_{2 m+1}, m=2,3, \cdots$, the forth length spectrum is $4 \pi \sqrt{\frac{3 m^{2}+9 m+7}{3\left(4 \kappa^{2}-c\right)}}$, the length of circles of complex torsion $\frac{\left(4 \kappa^{2}-c\right)^{3 / 2}}{3 \sqrt{3} c \kappa} \times$ $\tau(2 m+3,1)=\frac{9 m^{2}+27 m+7}{6 \sqrt{3} c \kappa} \times\left(\frac{4 \kappa^{2}-c}{3 m^{2}+9 m+7}\right)^{3 / 2}$.

Remark. For positive constants $\tau_{1}, \tau_{2}$ with $0 \leq \tau_{1}<\tau_{2} \leq \nu(\kappa)$ we put

$$
\operatorname{Cir}_{\kappa}^{\left(\tau_{1}, \tau_{2}\right)}\left(\mathbb{C} H^{n}(-c)\right)=\left\{\left[\gamma_{\kappa, \tau}\right] \mid \tau_{1}<\tau<\tau_{2}\right\}
$$


As we have

$$
\begin{aligned}
\mathcal{L}\left(\operatorname{Cir}_{\kappa}\left(\mathbb{C} P^{n}(c)\right)\right) \cap \mathbb{R} & \\
= & \bigcup\left\{4 \pi \sqrt{\frac{3 p^{2}+q^{2}}{3\left(4 \kappa^{2}-c\right)}} \begin{array}{l}
\beta_{2} q<p<\beta_{1} q, p q \text { is even, } \\
p \text { and } q \text { are mutually prime }
\end{array}\right\} \\
& \bigcup\left\{2 \pi \sqrt{\frac{3 p^{2}+q^{2}}{3\left(4 \kappa^{2}-c\right)}} \mid \begin{array}{l}
\beta_{2} q<p<\beta_{1} q, p q \text { is odd, } \\
p \text { and } q \text { are mutually prime }
\end{array}\right\},
\end{aligned}
$$

where $\beta_{i}(i=1,2)$ denote the positive numbers with $\frac{3 \sqrt{3} c \kappa \tau_{i}}{\left(4 \kappa^{2}-c\right)^{3 / 2}}=\frac{9 \beta_{i}^{2}-1}{\left(3 \beta_{i}^{2}+1\right)^{3 / 2}}$, we obtain that the set $\left\{\mathcal{L}\left(\left[\gamma_{\kappa, \tau}\right]\right) \mid\left[\gamma_{\kappa, \tau}\right] \in \operatorname{Cir}_{\kappa}^{\left(\tau_{1}, \tau_{2}\right)}\left(\mathbb{C} H^{n}(-c)\right)\right\} \backslash\{\infty\}$ is not

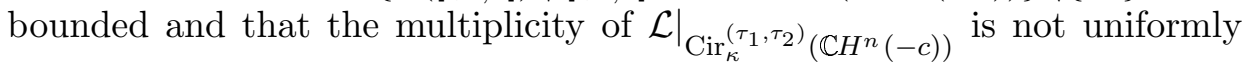
finite.

Summarizing up we obtain the following on the length spectrum of

\begin{tabular}{|c|c|c|}
\hline & $\mathbb{C} H^{n}(-c)$ & $\mathbb{C} P^{n}(c)$ \\
\hline $\operatorname{LSpec}_{\kappa}$ & $\begin{array}{l}\emptyset, \quad \text { if } 0 \leq \kappa \sqrt{c} / 2, \\
\text { discrete, unbounded, } \\
\text { if } \kappa>\sqrt{c} / 2\end{array}$ & discrete, unbounded \\
\hline multiplicity & $\begin{aligned} \limsup _{\lambda \rightarrow \infty} \sharp\left(\mathcal{L}_{\kappa}^{-1}(\lambda)\right)=\infty, & \\
& \text { if } \kappa>\sqrt{c} / 2\end{aligned}$ & $\limsup _{\lambda \rightarrow \infty} \sharp\left(\mathcal{L}_{\kappa}^{-1}(\lambda)\right)=\infty$ \\
\hline $\begin{array}{l}\text { 1st length } \\
\text { spectrum }\end{array}$ & $\begin{array}{l}\frac{4 \pi}{\sqrt{4 \kappa^{2}-c}}, \text { simple, if } \kappa>\sqrt{c} / 2 \\
\text { (length spectrum of } \\
\text { totally real circles) }\end{array}$ & $\begin{array}{l}\frac{2 \pi}{\sqrt{\kappa^{2}+c}}, \text { simple, } \\
\text { (length spectrum of } \\
\text { holomorphic circles) }\end{array}$ \\
\hline $\begin{array}{l}\text { 2nd length } \\
\text { spectrum }\end{array}$ & 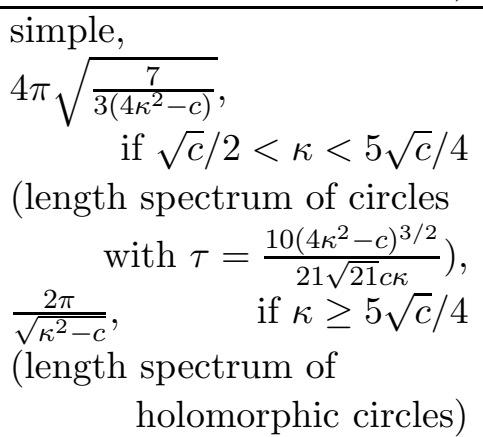 & $\begin{array}{l}\text { simple, } \\
\frac{4 \pi}{\sqrt{4 \kappa^{2}+c}} \\
\text { (length spectrum of } \\
\quad \text { totally real circles) }\end{array}$ \\
\hline
\end{tabular}
circles on non-flat complex space forms with geodesic curvature $\kappa$.

Table for length spectrum of circles of geodesic curvature $\kappa$ 


\section{$\S 4$. The full length spectrum}

In this section we study the full length spectrum $\mathcal{L}$ of circles on a complex hyperbolic space $\mathbb{C} H^{n}(-c)$ of holomorphic sectional curvature $-c$. Since the lengths of holomorphic circles and totally real circles of geodesic curvature $\kappa$ are $\frac{2 \pi}{\sqrt{\kappa^{2}-c}}$ and $\frac{4 \pi}{\sqrt{4 \kappa^{2}-c}}$ respectively, we see that both the length spectrum $\operatorname{LSpec}^{1}\left(\mathbb{C} H^{n}(-c)\right)$ of all holomorphic circles and the length spectrum $\operatorname{LSpec}^{0}\left(\mathbb{C} H^{n}(-c)\right)$ of all totally real circles coincide with the half line $(0, \infty)$. Therefore the multiplicity of $\mathcal{L}$ at $\lambda \in(0, \infty)$ is at least 2. This asserts that we can not distinguish any congruency classes of circles only by their length spectrum. This is a quite different point from the case of a complex projective space. On a complex projective space $\mathbb{C} P^{n}(c)$ of holomorphic sectional curvature $c$, the full length spectrum is simple at $\lambda \in\left(\frac{2}{\sqrt{c}} \pi, \frac{4}{3} \sqrt{\frac{5}{c}} \pi\right]$, which means that we can determine the congruency class only by the length spectrum if it is contained in this interval.

In order to investigate the length spectrum of circles with complex torsion $0<|\tau|<1$, we make use of the natural foliation of the moduli space of bounded circles. For given $\mu(>0)$ we denote by $K_{\mu}$ the positive solution for the equation $\left(4 \kappa^{2}-c\right)^{3 / 2} \mu-3 \sqrt{3} c \kappa=0$. In case $\mu>1$, we see that $K_{\mu}=\kappa\left(\frac{1}{\mu}\right)$ by using the notation in section 2. Clearly we find that $K_{\mu}>\sqrt{c} / 2$ and that $0<\frac{\left(4 \kappa^{2}-c\right)^{3 / 2} \mu}{3 \sqrt{3} c \kappa}<1$ when $\sqrt{c} / 2<\kappa<K_{\mu}$. When $\mu=\tau(p, q)=q\left(9 p^{2}-q^{2}\right)\left(3 p^{2}+q^{2}\right)^{-3 / 2}$, we can solve this equation and get $K_{\tau(p, q)}^{2}=\frac{c(3 p+q)^{2}}{8 q(3 p-q)}$, hence $\frac{3 p^{2}+q^{2}}{3\left(4 K_{\tau(p, q)}^{2}-c\right)}=\frac{2 q(3 p-q)}{9 c}$. By Lemmas 1 and 2 we obtain that



Here

$$
I_{\tau(p, q)}= \begin{cases}\left(\frac{4 \pi}{3} \sqrt{\frac{2 q(3 p-q)}{c}}, \infty\right), & \text { if } p q \text { is even } \\ \left(\frac{2 \pi}{3} \sqrt{\frac{2 q(3 p-q)}{c}}, \infty\right), & \text { if } p q \text { is odd }\end{cases}
$$

For example we have

$$
I_{\tau(2,1)}=\left(\frac{4}{3} \sqrt{\frac{10}{c}} \pi, \infty\right), I_{\tau(3,1)}=\left(\frac{8}{3 \sqrt{c}} \pi, \infty\right), I_{\tau(5,1)}=\left(\frac{4}{3} \sqrt{\frac{7}{c}} \pi, \infty\right) .
$$

Proposition 6. The multiplicity of length spectrum $\mathcal{L}$ of circles on $\mathbb{C} H^{n}(-c)$ is finite at each point $\lambda \in \mathbb{R}$, and is monotone increasing with 
respect to $\lambda$. There are no circles $\gamma$ of complex torsion $0<|\tau|<1$ with length $(\gamma) \leq \frac{8}{3 \sqrt{c}} \pi$

Proof. First we show that the multiplicity of $\mathcal{L}$ is finite at each point $\lambda \in(0, \infty)$. As we have $q(3 p-q) \geq 3 p-q>2 p$ for $p>q \geq 1$, we find that if $\lambda \in I_{\tau(p, q)}$ then $q<p<\frac{9 c \lambda^{2}}{16 \pi^{2}}$. Thus we obtain that the number of pairs $(p, q)$ of mutually prime positive numbers with $p>q$ and with $\lambda \in I_{\tau(p, q)}$ is finite for each $\lambda$. This leads us to that the multiplicity of $\mathcal{L}$ is finite at $\lambda$. The monotonous property of the multiplicity follows directly from the shape of $I_{\tau(p, q)}$.

In order to show the last assertion we are enough to prove that $I_{\tau(p, q)} \subset$ $\left(\frac{8}{3 \sqrt{c}} \pi, \infty\right)$. Since $q(3 p-q)>2 p \geq 2$, we have $\frac{8}{3 \sqrt{c}}<\frac{4 \pi}{3} \sqrt{\frac{2 q(3 p-q)}{c}}$, hence $I_{\tau(p, q)} \subset\left(\frac{8}{3 \sqrt{c}} \pi, \infty\right)$ when $p q=$ even. If $p q=$ odd and $q \geq 3$ we have $q(3 p-q) \geq 3(3 p-q)>6 p>24$, and if $p q=$ odd, $q=1$ and $p \geq 5$ we have $q(3 p-q)=3 p-1>8$. Therefore in these cases we get $\frac{8}{3 \sqrt{c}}<\frac{2 \pi}{3} \sqrt{\frac{2 q(3 p-q)}{c}}$, hence $I_{\tau(p, q)} \subset\left(\frac{8}{3 \sqrt{c}} \pi, \infty\right)$, and obtain the assertion.

By a similar argument we find that the multiplicity of $\mathcal{L}$ at $\lambda \in\left(\frac{8}{3 \sqrt{c}} \pi\right.$, $\left.\frac{4}{3} \sqrt{\frac{7}{c}} \pi\right]$ is 3 , at $\lambda \in\left(\frac{4}{3} \sqrt{\frac{7}{c}} \pi, \frac{4}{3} \sqrt{\frac{10}{c}} \pi\right]$ is 4 , and is greater than 5 at $\lambda>$ $\frac{4}{3} \sqrt{\frac{10}{c}} \pi$. Let $m_{\mathcal{L}}:[0, \infty) \rightarrow \mathbb{Z}$ denote the multiplicity function defined by $m_{\mathcal{L}}(\lambda)=\sharp \mathcal{L}^{-1}(\lambda)$. By the expression of $\operatorname{LSpec}\left(\mathbb{C} H^{n}(-c)\right)$ we find that this function is monotone increasing and left continuous. We can estimate $m_{\mathcal{L}}$ as $m_{\mathcal{L}}\left(\frac{4 \pi}{3} \sqrt{\frac{\lambda}{c}}\right)<\lambda(\lambda-1)+2$ by recalling the proof of the first assertion of Proposition 6. On the other hand, since $\frac{4 \pi}{3} \sqrt{\frac{\lambda}{c}} \in I_{\tau(p, 1)}$ provided $p<\frac{\lambda}{6}$, we obtain that $m_{\mathcal{L}}\left(\frac{4 \pi}{3} \sqrt{\frac{\lambda}{c}}\right)>\left[\frac{\lambda}{6}\right]+2$, where $[\alpha]$ denotes the integer part of a number $\alpha$. The set

$$
\begin{aligned}
&\{\rho \in \mathbb{R}\left.\mid \lim _{\lambda \downarrow \rho} m_{\mathcal{L}}(\lambda)-m_{\mathcal{L}}(\rho)>0\right\} \\
&=\left\{\frac{8 \pi}{3 \sqrt{c}}, \frac{4 \pi}{3 \sqrt{c}} \sqrt{7}, \frac{4 \pi}{3 \sqrt{c}} \sqrt{10}, \frac{4 \pi}{3 \sqrt{c}} \sqrt{14}, \frac{16 \pi}{3 \sqrt{c}}, \frac{4 \pi}{\sqrt{c}} \sqrt{2}\right. \\
&\left.\frac{4 \pi}{3 \sqrt{c}} \sqrt{19}, \frac{4 \pi}{3 \sqrt{c}} \sqrt{22}, \frac{20 \pi}{3 \sqrt{c}}, \frac{4 \pi}{\sqrt{c}} \sqrt{3}, \frac{8 \pi}{3 \sqrt{c}} \sqrt{7}, \frac{4 \pi}{3 \sqrt{c}} \sqrt{31}, \cdots\right\}
\end{aligned}
$$

of discontinuous points of $m_{\mathcal{L}}$ is discrete and unbounded. Moreover the jumping step $\lim _{\lambda \downarrow \rho} m_{\mathcal{L}}(\lambda)-m_{\mathcal{L}}(\rho)$ is not uniformly bounded with respect 
to $\rho$. The first two steps jumping point is $\frac{4 \pi}{3 \sqrt{c}} \sqrt{10}$ and the second is $\frac{8 \pi}{3 \sqrt{c}} \sqrt{7}$; $I_{\tau(2,1)}=I_{\tau(7,1)}=\left(\frac{4 \pi}{3 \sqrt{c}} \sqrt{10}, \infty\right)$ and $I_{\tau(3,2)}=I_{\tau(19,1)}=\left(\frac{8 \pi}{3 \sqrt{c}} \sqrt{7}, \infty\right)$. The jumping step at $4 \pi \sqrt{\frac{30}{c}}$ is at least $3 ; I_{\tau(61,3)}=I_{\tau(23,9)}=I_{\tau(17,15)}=$ $\left(4 \pi \sqrt{\frac{30}{c}}, \infty\right)$. In general, we can find a higher steps jumping point by putting $q=3, p=1+\Pi m_{j}, q_{i}=3 m_{i}$ and $p_{i}=m_{i}+\frac{p-1}{m_{i}}$, where $m_{j}$ are mutually distinct positive prime numbers; $I_{\tau(p, q)}=I_{\tau\left(p_{i}, q_{i}\right)}$.

The fact that the multiplicity of $\mathcal{L}$ at $\lambda \in\left(\frac{8}{3 \sqrt{c}} \pi, \frac{4}{3} \sqrt{\frac{7}{c}} \pi\right]$ is 3 guarantees that there are no circles $\gamma$ of complex torsion $0<\tau<\tau(3,1)=\frac{10}{7 \sqrt{7}}$ with $\operatorname{length}(\gamma) \leq \frac{4}{3} \sqrt{\frac{7}{c}} \pi$. More generally, we have the following:

Proposition 7. For each $\lambda(>4)$, we have no circle $\gamma$ on $\mathbb{C} H^{n}(-c)$ with length $(\gamma) \leq \frac{4 \pi}{3} \sqrt{\frac{\lambda}{c}}$ and of complex torsion $0<|\tau|<\frac{20}{\lambda \sqrt{\lambda+21}}$.

Proof. We shall show that the complex torsion of a circle $\gamma$ with length $(\gamma)=\frac{4 \pi}{3} \sqrt{\frac{\lambda}{c}}$ is not smaller than $\frac{20}{\lambda \sqrt{\lambda+21}}$. If the congruency class containing $\gamma$ lies in the leaf $\mathcal{F}_{\tau(p, q)}(p q=$ even $)$ of the natural foliation, its geodesic curvature $\kappa$ satisfies $\sqrt{\frac{3 p^{2}+q^{2}}{3\left(4 \kappa^{2}-c\right)}}=\frac{1}{3} \sqrt{\frac{\lambda}{c}}$, hence $\kappa=$ $\frac{1}{2} \sqrt{c\left\{1+\frac{3}{\lambda}\left(3 p^{2}+q^{2}\right)\right\}}$. Therefore the complex torsion is

$$
\mu(p, q)=\frac{\left(4 \kappa^{2}-c\right)^{3 / 2}}{3 \sqrt{3} c \kappa} \tau(p, q)=\frac{2 q\left(9 p^{2}-q^{2}\right)}{\lambda \sqrt{\lambda+3\left(3 p^{2}+q^{2}\right)}} .
$$

Since this constant is monotone increasing with respect $q(1 \leq q<p)$ for each $p$ and is monotone increasing with respect to $p$ for each $q$, we have that $\mu(p, q) \geq \mu(p, 1) \geq \mu(2,1)=\frac{70}{\lambda \sqrt{\lambda+39}}$ when $p q=$ even. Similarly if the congruency class containing $\gamma$ lies in the leaf $\mathcal{F}_{\tau(p, q)}(p q=$ odd), its geodesic curvature $\kappa$ satisfies $\sqrt{\frac{3 p^{2}+q^{2}}{3\left(4 \kappa^{2}-c\right)}}=\frac{2}{3} \sqrt{\frac{\lambda}{c}}$ and the complex torsion is

$$
\mu(p, q)=\frac{\left(4 \kappa^{2}-c\right)^{3 / 2}}{3 \sqrt{3} c \kappa} \tau(p, q)=\frac{q\left(9 p^{2}-q^{2}\right)}{2 \lambda \sqrt{4 \lambda+3\left(3 p^{2}+q^{2}\right)}} .
$$

We also find that $\mu(p, q) \geq \mu(3,1)=\frac{20}{\lambda \sqrt{\lambda+21}}$ when $p q=$ odd. Since $\lambda>4$ we have $\mu(3,1)<\mu(2,1)$ and get the conclusion. 
This proposition tells us that circles with small complex torsions have long prime periods.

Remark. By the proof of Proposition 7 we find the following: If a circle $\gamma$ has prime period $\frac{4 \pi}{3} \sqrt{\frac{\lambda}{c}}$ and is not neither holomorphic nor totally real then it has either

1) complex torsion $\mu(p, q)$ and geodesic curvature $\frac{1}{2} \sqrt{c\left\{1+\frac{3\left(3 p^{2}+q^{2}\right)}{\lambda}\right\}}$ for some mutually prime positive numbers $p, q$ with $p q=$ even, $p>q$ and with $2 q\left(9 p^{2}-q^{2}\right)<\lambda \sqrt{\lambda+3\left(3 p^{2}+q^{2}\right)}$, or

2) complex torsion $\mu(p, q)$ and geodesic curvature $\frac{1}{2} \sqrt{c\left\{1+\frac{3\left(3 p^{2}+q^{2}\right)}{4 \lambda}\right\}}$ for some $p, q$ with $p q=$ odd, $p>q$ and with $q\left(9 p^{2}-q^{2}\right)<$ $2 \lambda \sqrt{4 \lambda+3\left(3 p^{2}+q^{2}\right)}$.

Table for full length spectrum of circle

\begin{tabular}{|c|l|c|}
\hline & \multicolumn{1}{|c|}{$\mathbb{C}^{n}(-c)$} & \multicolumn{1}{c|}{$\mathbb{C} P^{n}(c)$} \\
\hline LSpec & \multicolumn{1}{|c|}{$(0, \infty)$} & $(0, \infty)$ \\
\hline & finite at each $\lambda$, & finite at each $\lambda$, \\
& monotone increasing, & \\
polynomial growth, & \\
multiplicity $m_{\mathcal{L}}$ & $\begin{array}{l}\text { left continuous, } \\
m_{\mathcal{L}}(\lambda) \geq 2,\end{array}$ & $m_{\mathcal{L}}(\lambda) \geq 1$, \\
& $m_{\mathcal{L}}(\lambda)=2$ if and only & $m_{\mathcal{L}}(\lambda)=1$ if and only \\
& if $0<\lambda \leq \frac{8 \pi}{3 \sqrt{c}}$ & if $\frac{2}{\sqrt{c}}<\lambda \leq \frac{4}{3} \sqrt{\frac{5}{c}} \pi$ \\
\hline
\end{tabular}

\section{$\S 5$. The restricted length spectrum of circles}

In order to get more information on the distribution of length spectrum, we shall study another restricted length spectrum, the length spectrum of circles of complex torsion $\tau$ on a complex hyperbolic space $\mathbb{C} H^{n}(-c)$. Let $\operatorname{Cir}^{\tau}\left(\mathbb{C} H^{n}(-c)\right)$ denote the moduli space of circles of complex torsion $\tau$, and $\mathcal{L}^{\tau}$ the restriction of $\mathcal{L}$ onto this space. We set $\operatorname{LSpec}^{\tau}\left(\mathbb{C} H^{n}(-c)\right)=$ $\mathcal{L}\left(\operatorname{Cir}^{\tau}\left(\mathbb{C} H^{n}(-c)\right)\right) \cap \mathbb{R}$. By Lemmas 1, 2 and Fact 2, we find for $0<\tau<1$ that

$$
\begin{aligned}
\operatorname{LSpec}^{\tau}\left(\mathbb{C} H^{n}(-c)\right) & \\
= & \bigcup\left\{4 \pi \sqrt{\frac{3 p^{2}+q^{2}}{3\left(4 K_{\tau(p, q) / \tau}^{2}-c\right)}} \mid \begin{array}{l}
p>q, p q \text { is even, } \\
p \text { and } q \text { are mutually prime }
\end{array}\right\} \\
& \bigcup\left\{2 \pi \sqrt{\frac{3 p^{2}+q^{2}}{3\left(4 K_{\tau(p, q) / \tau}^{2}-c\right)}} \mid \begin{array}{l}
p>q, p q \text { is odd }, \\
p \text { and } q \text { are mutually prime }
\end{array}\right\} .
\end{aligned}
$$


We first study set theoretical properties of $\operatorname{LSpec}^{\tau}\left(\mathbb{C} H^{n}(-c)\right)$.

TheOREM 4. For every $\tau(0<\tau<1)$, the length spectrum of circles $\operatorname{LSpec}^{\tau}\left(\mathbb{C} H^{n}(-c)\right)$ of complex torsion $\tau$ is a discrete unbounded subset of the real line.

Proof. First we show that $\operatorname{LSpec}^{\tau}\left(\mathbb{C} H^{n}(-c)\right)$ is unbounded. We set $K_{n}=K_{\tau(n+1, n) / \tau}$, which satisfies that $3 \sqrt{3} c \tau K_{n}\left(4 K_{n}^{2}-c\right)^{-3 / 2}=\tau(n+1, n)$. Since $\lim _{n \rightarrow \infty} \tau(n+1, n)=1$ we find $\lim _{n \rightarrow \infty} K_{n}=\kappa(\tau)$. Hence we have

$$
\mathcal{L}\left(\left[\gamma_{K_{n}, \tau}\right]\right)=\sqrt{\frac{3 c}{4 K_{n}^{2}-c}} \times \mathcal{L}\left(\left[\gamma_{\sqrt{c}, \tau(n+1, n)}\right]\right)=4 \pi \sqrt{\frac{3(n+1)^{2}+n^{2}}{3\left(4 K_{n}^{2}-c\right)}} \longrightarrow \infty
$$

and get that the set $\operatorname{LSpec}^{\tau}\left(\mathbb{C} H^{n}(-c)\right)$ is not bounded.

Next we show that $\operatorname{LSpec}^{\tau}\left(\mathbb{C} H^{n}(-c)\right)$ is discrete. For given $\kappa\left(>\frac{\sqrt{c}}{2}\right)$ we put $\mu(\kappa)=3 \sqrt{3} c \kappa \tau\left(4 \kappa^{2}-c\right)^{-3 / 2}$. If we suppose $\operatorname{LSpec}^{\tau}\left(\mathbb{C} H^{n}(-c)\right)$ has an accumulation point $\xi \in \mathbb{R}$, then there exists a sequence $\left\{\kappa_{n}\right\}_{n=1}^{\infty}$ of positive numbers with

$$
\mathcal{L}\left(\left[\gamma_{\kappa_{n}, \tau}\right]\right)<\infty, \xi=\lim _{n \rightarrow \infty} \mathcal{L}\left(\left[\gamma_{\kappa_{n}, \tau}\right]\right)
$$

By Lemmas 1 and 2 , we find that $\mu\left(\kappa_{n}\right)$ is expressed as $\mu\left(\kappa_{n}\right)=q_{n}\left(9 p_{n}^{2}-\right.$ $\left.q_{n}^{2}\right)\left(3 p_{n}^{2}+q_{n}^{2}\right)^{-3 / 2}$ by mutually prime positive integers $p_{n}, q_{n}\left(p_{n}>q_{n}\right)$.

When the set $\left\{\kappa_{n} \mid n=1,2, \cdots\right\}$ is unbounded, by taking a subsequence, we may suppose $\lim _{n \rightarrow \infty} \kappa_{n}=\infty$. In this case we can conclude that $\lim _{n \rightarrow \infty} \frac{p_{n}}{q_{n}}=\infty$ and

$$
\lim _{n \rightarrow \infty} \frac{p_{n}}{\kappa_{n}^{2} q_{n}}=\lim _{n \rightarrow \infty} \frac{\mu\left(\kappa_{n}\right)\left(\frac{p_{n}}{q_{n}}\right)}{\mu\left(\kappa_{n}\right) \kappa_{n}^{2}}=\frac{8}{3 c \tau}(<\infty) .
$$

We therefore get

$$
\mathcal{L}\left(\left[\gamma_{\kappa_{n}, \tau}\right]\right)=\sqrt{\frac{3 c}{\left(4 \kappa_{n}^{2}-c\right)}} \times \mathcal{L}\left(\left[\gamma_{\sqrt{c}, \mu\left(\kappa_{n}\right)}\right]\right) \geq 2 \pi \sqrt{\frac{3 p_{n}^{2}+q_{n}^{2}}{3\left(4 \kappa_{n}^{2}-c\right)}} \longrightarrow \infty .
$$

This contradicts to the assumption $\xi<\infty$.

When $\left\{\kappa_{n} \mid n=1,2, \cdots\right\}$ is a bounded set we see that $\left\{\sqrt{\frac{3 c}{4 \kappa_{n}^{2}-c}} \mid n=\right.$ $1,2, \cdots\}$ is bounded from below. Hence we find the set $\left\{\mathcal{L}\left(\left[\gamma_{\frac{\sqrt{2 c}}{4}, \mu\left(\kappa_{n}\right)}\right]\right) \mid\right.$ $n=1,2, \cdots\}$ is also bounded. Since the set

$$
\left\{\begin{array}{l|l}
(p, q) & \begin{array}{l}
p \text { and } q \text { are mutually prime positive } \\
\text { numbers, } p>q, \sqrt{3 p^{2}+q^{2}} \leq L
\end{array}
\end{array}\right\}
$$


is a finite set for each $L$, we find the set $\left\{\kappa_{n} \mid n=1,2, \cdots\right\}$ is also a finite set, which is a contradiction. Thus we obtain that $\left.\operatorname{LSpec}^{\tau}\left(\mathbb{C} H^{n}(-c)\right)\right)$ is a discrete set.

Remark. Put $\operatorname{Cir}_{\left(\kappa_{1}, \kappa_{2}\right)}^{\tau}\left(\mathbb{C} H^{n}(-c)\right)=\left\{\left[\gamma_{\kappa, \tau}\right] \mid \kappa_{1}<\kappa<\kappa_{2}\right\}$ for $\kappa_{1}$ and $\kappa_{2}$ with $\kappa(\tau) \leq \kappa_{1}<\kappa_{2}<\infty$. We find that the set $\mathcal{L}\left(\operatorname{Cir}_{\left(\kappa_{1}, \kappa_{2}\right)}^{\tau}\left(\mathbb{C} H^{n}(-c)\right)\right) \cap$ $\mathbb{R}$ is also unbounded, because

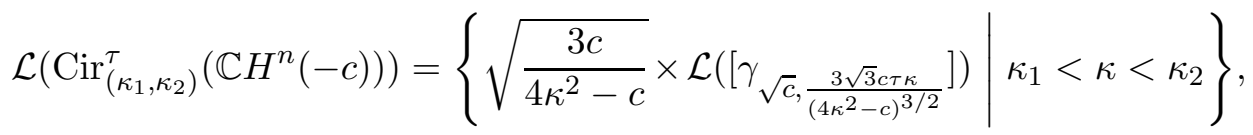

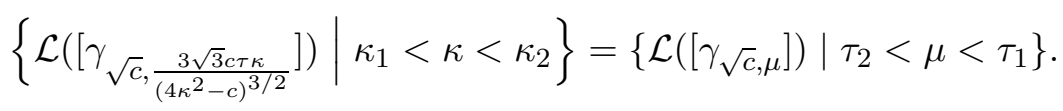

We now study the structure of $\operatorname{LSpec}^{\tau}\left(\mathbb{C} H^{n}(-c)\right)$.

Proposition 8. For every $\tau(0<\tau<1)$, the bottom of the length spectrum $\operatorname{LSpec}^{\tau}\left(\mathbb{C} H^{n}(-c)\right)$ is $4 \sqrt{7} \pi / \sqrt{3\left(4 K_{\tau(3,1) / \tau}^{2}-c\right)}$. This spectrum is simple for $\mathcal{L}^{\tau}$.

Proof. We put $K_{0}=K_{\tau(3,1) / \tau}$, which satisfies $\frac{100}{7^{3} \tau^{2}}\left(4 K_{0}^{2}-c\right)^{3}=27 c^{2} K_{0}^{2}$. Clearly by Lemma 1 we see that the length of a circle of geodesic curvature $K_{0}$ and complex torsion $\tau$ is $4 \sqrt{7} \pi / \sqrt{3\left(4 K_{\tau(3,1) / \tau}^{2}-c\right)}$. We shall show for mutually prime positive numbers $p, q$ with $p>q$ that

$$
4 \pi \sqrt{\frac{3 p^{2}+q^{2}}{3\left(4 K_{\tau(p, q) / \tau}^{2}-c\right)}}>4 \pi \sqrt{\frac{7}{3\left(4 K_{\tau(3,1) / \tau}^{2}-c\right)}}
$$

if $p q=$ even,

$$
2 \pi \sqrt{\frac{3 p^{2}+q^{2}}{3\left(4 K_{\tau(p, q) / \tau}^{2}-c\right)}}>4 \pi \sqrt{\frac{7}{3\left(4 K_{\tau(3,1) / \tau}^{2}-c\right)}}
$$

if $p q=$ odd and $(p, q) \neq(3,1)$.

Given $\mu(>0)$ we define a function $f_{\mu}:[\sqrt{c} / 2, \infty) \longrightarrow \mathbb{R}$ by $f_{\mu}(x)=$ $\mu^{2}(4 x-c)^{3}-27 c^{2} x$. Clearly $f_{\mu}\left(K_{\mu}^{2}\right)=0$, and $f_{\mu}(x)>0$ if and only if 
$x>K_{\mu}^{2}$. When $p q=$ even we have

$$
\begin{aligned}
f_{\tau(p, q) / \tau}\left(\frac{1}{4}\left\{\frac{1}{7}\left(3 p^{2}+q^{2}\right)\left(4 K_{0}^{2}-c\right)+c\right\}\right) \\
=\frac{1}{7^{3} \tau^{2}} q^{2}\left(9 p^{2}-q^{2}\right)^{2}\left(4 K_{0}^{2}-c\right)^{3}-\frac{27}{4} c^{2}\left\{\frac{1}{7}\left(3 p^{2}+q^{2}\right)\left(4 K_{0}^{2}-c\right)+c\right\} \\
=\frac{27 c^{2}}{2800}\left\{\left\{7 q^{2}\left(9 p^{2}-q^{2}\right)^{2}-100\left(3 p^{2}+q^{2}\right)\right\} K_{0}^{2}+25\left(3 p^{2}+q^{2}-7\right) c\right\} \\
>\frac{27 c^{2}}{700}\left\{7\left(8 p^{2}\right)^{2}-400 p^{2}\right\} K_{0}^{2} \\
>0
\end{aligned}
$$

because $p>q>0$. This guarantees that $4 K_{\tau(p, q) / \tau}^{2}<\frac{1}{7}\left(3 p^{2}+q^{2}\right)\left(4 K_{0}^{2}-\right.$ $c)+c$ in this case. When $p q=$ odd and $(p, q) \neq(3,1)$ we have

$$
\begin{aligned}
f_{\tau(p, q) / \tau} & \left(\frac{1}{4}\left\{\frac{1}{28}\left(3 p^{2}+q^{2}\right)\left(4 K_{0}^{2}-c\right)+c\right\}\right) \\
= & \frac{1}{4^{3} \times 7^{3} \tau^{2}} q^{2}\left(9 p^{2}-q^{2}\right)^{2}\left(4 K_{0}^{2}-c\right)^{3} \\
& -\frac{27}{4} c^{2}\left\{\frac{1}{28}\left(3 p^{2}+q^{2}\right)\left(4 K_{0}^{2}-c\right)+c\right\} \\
= & \frac{27 c^{2}}{44800}\left\{7 q^{2}\left(9 p^{2}-q^{2}\right)^{2}-1600\left(3 p^{2}+q^{2}\right)\right\} K_{0}^{2} \\
& +\frac{27 c^{3}}{448}\left(3 p^{2}+q^{2}-28\right) \\
> & 0 .
\end{aligned}
$$

Hence we have $4 K_{\tau(p, q) / \tau}^{2}<\frac{1}{28}\left(3 p^{2}+q^{2}\right)\left(4 K_{0}^{2}-c\right)+c$ in this case. Thus we obtain the desirable inequalities, we get the assertion.

Remark. When $\tau=\tau(3,1)$, the bottom is $\frac{4 \pi}{3} \sqrt{\frac{7}{c}}$. Since we can roughly estimate $K_{\mu}$ as

$$
\begin{aligned}
\frac{c}{4}\left(\frac{3}{\mu}+1\right)<K_{\mu}^{2}<\frac{c}{4}\left(\frac{3}{\sqrt{\mu}}+1\right) & \text { if } \mu>1, \\
\frac{c}{4}\left(\frac{3}{\sqrt{\mu}}+1\right)<K_{\mu}^{2}<\frac{c}{4}\left(\frac{3}{\mu}+1\right) & \text { if } \mu<1,
\end{aligned}
$$

we obtain the following rough estimates on the bottom of the length spec- 
trum $\operatorname{LSpec}^{\tau}\left(\mathbb{C} H^{n}(-c)\right):$ When $\tau>\tau(3,1)=\frac{10}{7 \sqrt{7}}$

$$
\frac{4 \pi}{3 \sqrt{c}} \times\left(\frac{100}{7 \tau^{2}}\right)^{\frac{1}{4}}<4 \pi \sqrt{\frac{7}{3\left(4 K_{\tau(3,1) / \tau}^{2}-c\right)}}<\frac{4 \pi}{3 \sqrt{c}} \times\left(\frac{10 \sqrt{7}}{\tau}\right)^{\frac{1}{4}}
$$

and when $\tau<\tau(3,1)$,

$$
\frac{4 \pi}{3 \sqrt{c}} \times\left(\frac{10 \sqrt{7}}{\tau}\right)^{\frac{1}{4}}<4 \pi \sqrt{\frac{7}{3\left(4 K_{\tau(3,1) / \tau}^{2}-c\right)}}<\frac{4 \pi}{3 \sqrt{c}} \times\left(\frac{100}{7 \tau^{2}}\right)^{\frac{1}{4}} .
$$

By the proof of Proposition 7, we can rewrite the bottom of the length spectrum $\operatorname{LSpec}^{\tau}\left(\mathbb{C} H^{n}(-c)\right)$ as $\frac{4 \pi}{3} \sqrt{\frac{\lambda}{c}}$ with $\lambda$ which satisfies $\tau^{2}\left(\lambda^{3}+21 \lambda^{2}\right)-$ $400=0$. By applying the same argument as in the proof of Proposition 8 , we find that the second and third length spectrum in $\operatorname{LSpec}^{\tau}\left(\mathbb{C} H^{n}(-c)\right)$ $(0<\tau<1)$ are $4 \pi \sqrt{19 / 3\left(4 K_{\tau(5,1) / \tau}^{2}-c\right)}$ and $4 \pi \sqrt{13 / 3\left(4 K_{\tau(2,1) / \tau}^{2}-c\right)}$, which are simple.

In the last stage we shall pose the following questions:

1) Are there non-simple spectrum in $\operatorname{LSpec}^{\tau}\left(\mathbb{C} H^{n}(-c)\right)$ ?

2) If such spectrum exist, how are their multiplicity? Is it true that the multiplicity of $\mathcal{L}^{\tau}(0<\tau<1)$ is not uniformly bounded?

The multiplicity of $\mathcal{L}^{\tau}$ at $\frac{4 \pi}{3} \sqrt{\frac{\lambda}{c}}$ equals to the cardinality of the following set of mutually prime positive numbers:

$$
\begin{aligned}
& \left\{(p, q) \mid p>q, p q=\text { even, } \frac{2 q\left(9 p^{2}-q^{2}\right)}{\lambda \sqrt{\lambda+3\left(3 p^{2}+q^{2}\right)}}=\tau\right\} \\
& \qquad\left\{(p, q) \mid p>q, p q=\text { odd }, \frac{q\left(9 p^{2}-q^{2}\right)}{2 \lambda \sqrt{4 \lambda+3\left(3 p^{2}+q^{2}\right)}}=\tau\right\} .
\end{aligned}
$$

\begin{tabular}{|c|c|c|}
\hline & $\mathbb{C} H^{n}(-c)$ & $\mathbb{C} P^{n}(c)$ \\
\hline $\begin{array}{l}\text { length spectrum of } \\
\text { holomorphic circles LSpec }\end{array}$ & $(0, \infty)$ & $\left(0, \frac{2 \pi}{\sqrt{c}}\right]$ \\
\hline $\begin{array}{l}\text { length spectrum of totally } \\
\text { real circles LSpec }\end{array}$ & $(0, \infty)$ & $\left(0, \frac{4 \pi}{\sqrt{c}}\right)$ \\
\hline $\operatorname{LSpec}^{\tau},(0<\tau<1)$ & $\begin{array}{c}\text { unbounded } \\
\text { discrete }\end{array}$ & $\begin{array}{c}\text { unbounded } \\
\text { discrete }\end{array}$ \\
\hline
\end{tabular}

Thus our problems are reduced to a problem on numbers.

Table for length spectrum of cirlces of complex torsion $\tau$ 


\section{REFERENCES}

[1] T. Adachi, Kähler magnetic flows on a manifold of constant holomorphic sectional curvature, Tokyo J. Math., 18 (1995), 473-483.

[2] T. Adachi, Circles on a quaternionic space form, J. Math. Soc. Japan, 48 (1996), 205-227.

[3] T. Adachi, A comparison theorem for magnetic Jacobi fields, Proc. Edinburgh Math. Soc., 40 (1997), 293-308.

[4] T. Adachi and S. Maeda, Global behaviours of circles in a complex hyperbolic spaces, Tsukuba J. Math., 21 (1997), 29-42.

[5] T. Adachi and S. Maeda, Length spectrum of circles in a complex projective spaces, Osaka J. Math., 35 (1998), 553-565.

[6] T. Adachi, S. Maeda and S. Udagawa, Circles in a complex projective spaces, Osaka J. Math., 32 (1995), 709-719.

[7] T. Adachi, S. Maeda and S. Udagawa, Circles in symmetric spaces are not necessarily simple, preprint.

[8] T. Adachi and T. Sunada, Twisted Perron-Frobenius theorem and L-functions, J. Func. Anal., 71 (1987), 1-46.

[9] A. L. Besse, Manifolds all of whose geodesics are closed, Springer-Verlag, Berlin Heidelberg New York, 1978.

[10] H. Busemann, The Geometry of Geodesics, Academic Press, New York, 1955.

[11] L. E. Dikson, History of the Theory of Numbers III, Quadratic and higer forms, Chelsea Publishing Company, New York, 1952.

[12] L. W. Green, Auf Wiedersehensflächen, Ann. of Math., 78 (1963), 289-299.

[13] G. H. Hardy, E. M. Wright, An introduction tothe theory of numbers, 4th edition, Oxford at the Clarendon Press, 1975.

[14] A. Katsuda and T. Sunada, Closed orbits in homology classes, Inst. Hautes Études Sci. Publ. Math., 71 (1990), 5-32.

[15] S. Maeda and Y. Ohnita, Herical geodesic immersion into complex space forms, Geom. Dedicata, 30 (1989), 93-114.

[16] K. Mashimo and K. Tojo, Circles in Riemannian symmetric spaces, preprint.

[17] K. Nomizu and K. Yano, On circles and spheres in Riemannian geometry, Math. Ann., 210 (1974), 163-170.

[18] W. Parry and M. Pollicott, An analogue of the prime number theorem for closed orbits of Axiom A flows, Ann. of Math., 118 (1983), 573-591.

[19] T. Sunada, Magnetic flows on a Riemann surface, Proc. KAIST Math. Workshop, 8 (1993, "Analysis and geometry"), 93-108.

[20] O. Zoll, Über Flächen mit Scharen geschlossener geodätischer Linien, Math. Ann., 57 (1903), 108-133.

Department of Mathematics

Nagoya Institute of Technology

Gokiso, Showa-ku, Nagoya 466-8555

Japan

adachi@math.kyy.nitech.ac.jp 